\title{
Auto Detection of Parkinson's Disease based on Objective Measurement of Gait Parameters using Wearable Sensors
}

\author{
Satyabrata $\mathrm{Aich}^{1 *}$ and Hee-Cheol Kim ${ }^{2}$ \\ ${ }^{1}$ Department of Computer Engineering, Inje University, Gimhae, S. Korea \\ ${ }^{2}$ Institute of Digital Anti-Aging Healthcare/Department of Computer \\ Engineering/Smart Wellness Lab, Inje University, Gimhae, S. Korea \\ ${ }^{1}$ satyabrataaich@gmail.com, ${ }^{2}$ heeki@inje.ac.kr
}

\begin{abstract}
Neurological diseases such as Parkinson's disease (PD) are becoming a major problem worldwide due to increasing longevity and the resulting aging population. Since $P D$ is progressive in nature, subjective assessment and monitoring of PD patients at different stages is cumbersome and time consuming. The innovation of low-cost wearable sensors and their suitability for objective gait parameter measurements facilitate the personalization of clinical treatment. With the development of machine learning techniques, it has also become possible to detect PD automatically based on gait parameters. In this study, we proposed a method for measuring gait parameters using wearable sensors and identified PD patients automatically based on machine learning techniques. The subjects of this study were 40 patients with PD and 40 control patients; the experimental set-up included three-dimensional (3D) motion analysis. Wearable devices were placed on the knees and ankles. Spatiotemporal gait parameter data were collected via 3D motion analysis. A performance comparison was conducted using different classification techniques with reduced feature sets obtained by the random feature elimination and principal component analysis methods. We obtained a maximum accuracy of $88.89 \%$ using a support vector machine with a radial basis function combined with a random feature elimination set. Our results demonstrate that the proposed technique can assist medical practitioners to distinguish PD patients from control groups using gait data. This method is recommended to medical practitioners for clinical implementation to personalize the monitoring and treatment of PD at different stages.
\end{abstract}

Keywords: Gait, machine learning, monitoring, Parkinson's disease, wearable sensors

\section{Introduction}

Parkinson's disease (PD) is among the most progressive neurodegenerative disorders among elderly patients and is characterized by motor and non-motor symptoms. Behaviors associated with PD include cognitive impairment, autonomic dysfunction, and neurobehavioral disorders [1]. In 2009, the World Health Organization reported that approximately 4 million people had been affected by PD [2]. To date, PD rates in Asian countries have not been evaluated in detail [3, 4]. Previous studies have reported that gait is an important predictor of various neurological diseases including PD [5]. The invention of inexpensive wearable devices and machine learning techniques have led to a decrease in the time and cost required for gait measurements and pattern recognition by clinicians. As the global population ages due to increased longevity, it is necessary to develop improved techniques to detect PD progression at different stages. In the past few years,

Received (April 16, 2018), Review Result (June 2, 2018), Accepted (June 9, 2018) 
artificial intelligence techniques have played an important role in gait classification and the diagnosis of diseases [6]. This study proposes the development of a new machine-learning-based tool and discusses its impact on successful PD detection and monitoring.

The structure of the paper is organized as follows: Section 2 presents the background. Section 3 describes about the methodology. Section 4 describes about the result and discussions. Section 5 describes about conclusion.

\section{Background}

Because PD affects mainly the elderly population, PD is becoming a challenge for the health care systems of developed countries and requires immediate solutions. Previous studies have found that wearable devices to measure gait parameters can contribute to monitoring PD at various stages. Schlachetzki et al., [7] used wearable sensors to quantify differences in gait parameters between PD patients and a control group of healthy elderly subjects. Sensors were attached to both shoes of the subjects in the PD and control groups, and gait impairment was detected in PD patients [7].

Sijobert et al., [8] explored the use of an inertial measurement unit, a system incorporating a gyroscope and an accelerometer, to conduct a gait analysis of healthy subjects and PD patients. They proposed an algorithm to estimate stride length using the data collected from the inertial measurement unit and validated their results by measuring stride length using GAITRite walkway system data, which is considered the gold standard. A comparison of algorithm and GAITRite system results showed that the mean error for all stride lengths was approximately $6 \%$ for healthy subjects and $10.3 \%$ for PD patients [8]. Pastorino et al., [9] used the PERFORM system, a tool for continuous monitoring of patients with neurodegenerative diseases, including $\mathrm{PD}$, and Zigbee protocols for data transmission. The PERFORM system uses triaxial accelerometers to measure limb acceleration, and an accelerometer and gyroscope are used to measure body acceleration and angular rate. Pastorino et al. determined that these wearable sensors allow clinicians to quantify motor fluctuations objectively [9].

Carcreff et al., [10] proposed a method to measure the spatiotemporal gait parameters of children with cerebral palsy, using wearable sensors and a signal processing algorithm. The proposed method provided good results, which were validated by comparison with those of the gold-standard optoelectronic system [10]. Adam et al., [11] conducted a pilot survey on the feasibility of wearable sensors for detecting different activity profiles among PD patients, Huntington's disease patients, and healthy subjects. They measured the subjects' activity levels during four activities (lying down, sitting, standing, and walking) and found significant differences among the activities, demonstrating the feasibility of wearable sensor data in clinical practice [11]. Suppa et al., [12] proposed a wearable system to distinguish PD patients experiencing falling of fait (FoG) episodes from those who had no FoG episodes. They found that the system was successful in detecting FoG episodes, with $93.41 \%$ sensitivity, $98.51 \%$ specificity, $89.55 \%$ positive predictive value (PPV), 97.31\% negative predictive value (NPV), and $98.51 \%$ accuracy. The authors validated their findings using video recordings and recommended this method for clinical practice [12]. Ilias et al., [13] assessed different motor states of patients in advanced stages of PD using wearable sensors with an accelerometer and gyroscope for data collection; data features were extracted using a time series-based method. The authors used a score-based method to differentiate motor states and found that upper limb sensors were good predictors of different stages of PD. There 
was a strong correlation between objective evaluations based on sensor data and subjective assessments by experts [13].

Several studies have implemented machine-learning-based approaches to examine and classify gait data. Prasertsung and Horanont [14] successfully classified pedestrian movements as walking along a normal path, climbing stairs, or walking down stairs, with an accuracy of $95.86 \%$ for training data and $85.73 \%$ for test data. Begg and Kamruzzaman [15] explored automated recognition of movement patterns by considering the basic, kinematic, and kinetics features of gait data and they have used SVM classifier and found accuracy of 91.7 while distinguishing two gait patterns. Hsu et al., [16] automated gait analysis using wearable sensors and found that a wearable instrument with an appropriately designed system to quantify gait parameters permitted early PD diagnosis. Tahir and Manap [17] used an artificial neural network (ANN) and support vector machine (SVM) to distinguish walking patterns during speed walking in PD patients by examining spatiotemporal, kinematic, and kinetic parameters. They observed perfect classification using the spatiotemporal parameter as a feature vector with an ANN and SVM classifier. Froelich et al., [18] diagnosed PD based on the characteristic features of a subject's voice using decision-tree-based classification with a threshold value. A mixed classification approach was applied to predict PD using nonlinear classifiers, with a classification accuracy of $90 \%$.

Li et al., [19] used a fuzzy transformation system to extract features from gait data, followed by principal component analysis (PCA) to determine the optimal features. Finally, they used an SVM to predict PD. Shahbakhi et al., [20] diagnosed PD based on speech analysis using a genetic algorithm and SVM, with accuracies of $94.50 \%, 93.66 \%$, and $94.22 \%$ for 4,7 , and 9 optimized features, respectively. Pogorelc et al., [21] examined a method to recognize health problems automatically using gait data with machine learning techniques. They applied an ANN with semantic features and a k-nearest neighbor classifier to gait data from subjects in different groups including hemiplegic, PD, back pain, leg pain, and normal. They obtained classification accuracies ranging from 99 to $100 \%$. Mansu and Hyunjin [22] distinguished scans without evidence of a dopaminergic deficit patients from PD patients by applying SVM classification to connectivity information derived from diffusion tensor imaging tractography and achieved $77.92 \%$ accuracy. Yi and $\mathrm{Yu}$ [23] used accelerometer and gyroscope data to develop a fall detection algorithm with $100 \%$ sensitivity and $98.75 \%$ specificity. Youn et al., [24] conducted a classification study to distinguish between mature and immature gait groups and obtained $81 \%$ accuracy using Waikato Environment for Knowledge Analysis tools. Prashanth and Roy [25] estimated different stages of PD using machine learning and clinical scales and found that the Movement Disorder Society-Unified Parkinson's Disease Rating Scale (MDS-UPDRS) was unsuitable for estimating the PD stage, although it does evaluate those features most closely related to PD. In contrast, the Hoehn and Yahr scale allowed PD stage classification but not evaluation of the features most closely related to PD. In that study, both the MDS-UPDRS and Hoehn and Yahr scale were used to evaluate stage classification by machine learning models with the SVM, ordinal logistic regression, and Adaboost and RUSBoostbased classifiers, obtaining $97.46 \%$ accuracy with the AdaBoost ensemble-based classifier. MDS-UPDRS with classifiers was deemed an important tool to predict different PD stages and thus may improve diagnostic systems. Abdulhay et al., [26] investigated gait and tremors using machine-learning-based techniques for the treatment of PD and developed a pulse duration algorithm to detect different temporal features. They used an SVM-based classification method and obtained an average accuracy of $92.7 \%$ and an area under the curve of $97 \%$. The method 
developed in that study is effective in determining PD treatment using gait and tremor analysis.

\section{Methodology}

This study included a total of 80 individuals: 40 PD patients and 40 patients in a control group. We collected gait data for 12 spatiotemporal parameters such as cadence(C), stride time(ST), opposite foot off(OFO), opposite foot contact(OFC), step time(SPT), single support(SS), double support(DS), foot off(FO), stride length(SL), step length(SPL), walking speed(WS), step width(SW) from these individuals using wearable devices and a three-dimensional (3D) motion analysis system at Inje Paik Hospital, Busan, South Korea. We used two feature-selection methods: random feature elimination (RFE) and PCA. The RFE selection method is a recursive process that ranks features according to a measure of their importance [27]. PCA is used to compress data and extract information [28]. Figure 1 shows a flow chart of the methodology used in this study.

\subsection{Classification Methods}

We used a nonlinear classifier with a decision tree for classification into the following groups: recursive partitioning and regression trees(RPART), C4.5, pruning rule-based classification tree(PART), bagging classification and regression tree, Random Forest and Boosted C5.0 with the Naïve Bayes method as the probabilistic classifier, and LDA with the SVM and radial basis function (RBF) as linear classifiers.

\subsection{Performance Measure Metrics}

The parameters used to assess and validate the classifiers were as follows: accuracy, sensitivity, specificity, PPV, and NPV. Sensitivity is defined as the ratio of true positives to the sum of true positives and false negatives. Specificity is the ratio of true negatives to the sum of false positives and true negatives. In the current study, we used the PPV and NPV to verify the presence or absence of PD. Therefore, PPV represents the probability that PD is present given a positive test result, and NPV is the probability that PD is absent given a negative test result [29]. Accuracy is defined as the ratio of the number of correct predictions to the total number of predictions, multiplied by 100 to convert to a percentage.

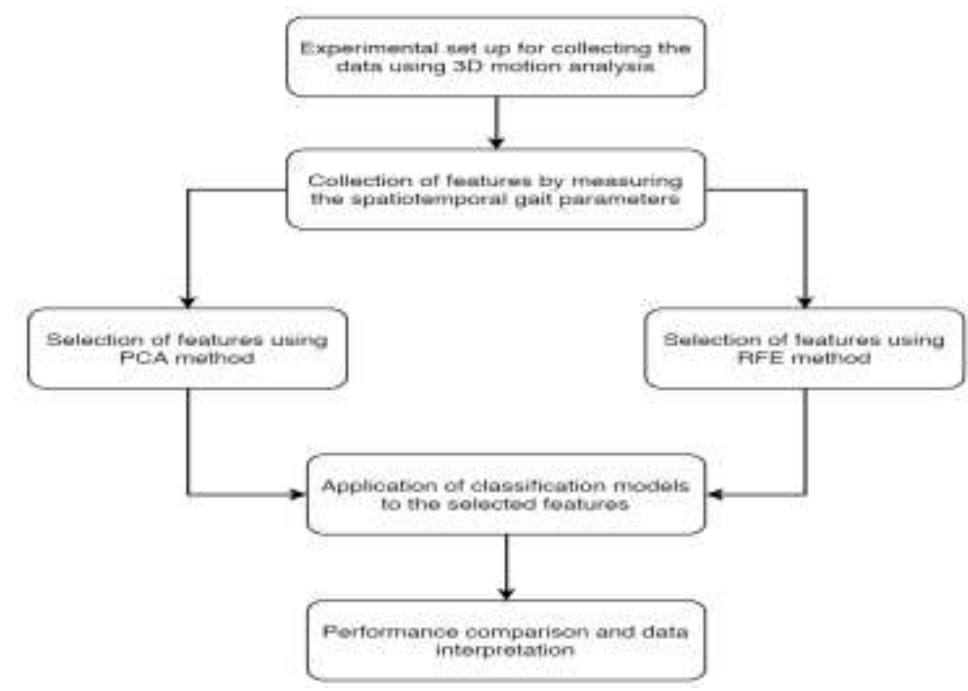

Figure 1. Flow Chart of the Proposed Methodology 


\section{Result and Discussions}

The original feature sets consisted of 12 features, selected based on the RFE and PCA methods (Table 1). For the RFE method, we selected features with the subset of attributes that provided the best comparison results with respect to all attributes. PCA is a dimension reduction technique, and the principal components are the directions in which variance is greatest, i.e., greater dispersion. Figure 2 shows the selected features obtained by the RFE method; the 11 selected features were able to provide the same accuracy as the 12 original features. Figure 3 shows the variance of the selected variables and their contributions to feature selection; six variables accounted for a variance of $98.5 \%$. Figure 4 shows the contributions of the variables obtained by PCA to the feature selection process. Both of these feature-selection techniques are used to identify a small number of important features and to compare the performance of the system in response to these features.

\section{Table 1. Feature Selection using Different Methods}

\begin{tabular}{|l|c|}
\hline Method & Feature \\
\hline RFE & $\begin{array}{c}\text { Cadence, stride time, opposite foot off, steptime, single support, } \\
\text { double support, foot off, stride length, step length, walking } \\
\text { speed, step width }\end{array}$ \\
\hline PCA & $\begin{array}{c}\text { Stride time, step time, single support, stride length, step length, } \\
\text { walking speed }\end{array}$ \\
\hline
\end{tabular}

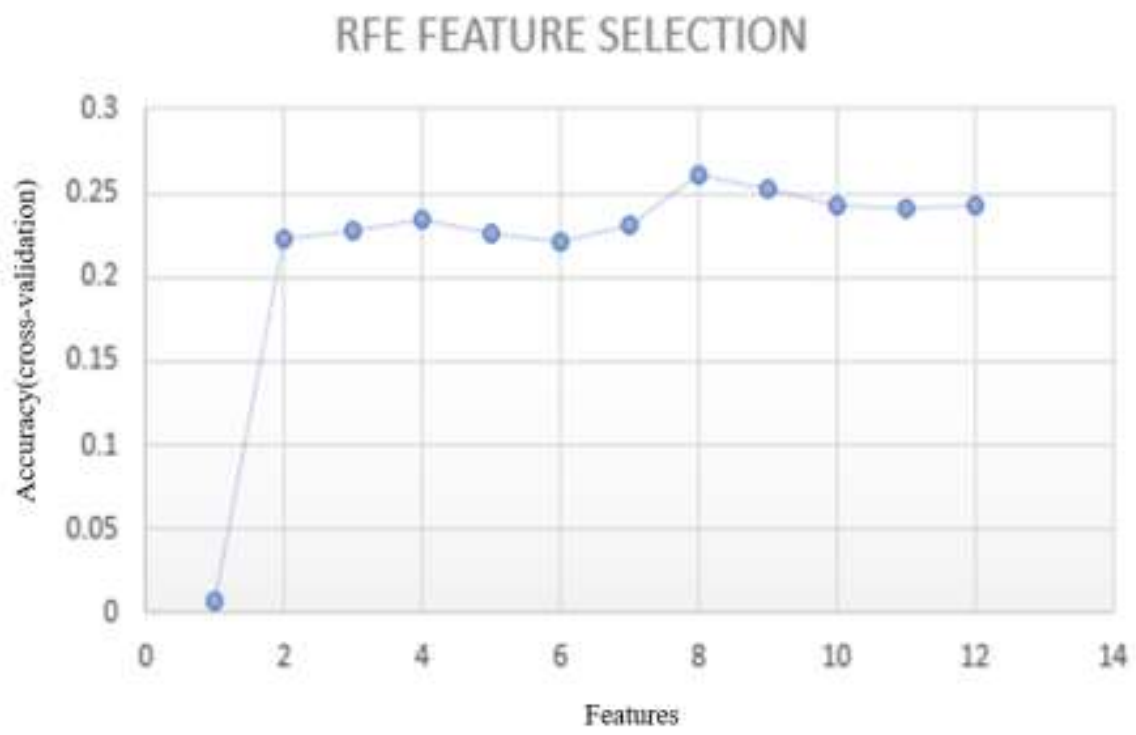

Figure 2. Features Selected Features based on the Random Feature Elimination (RFE) Method 


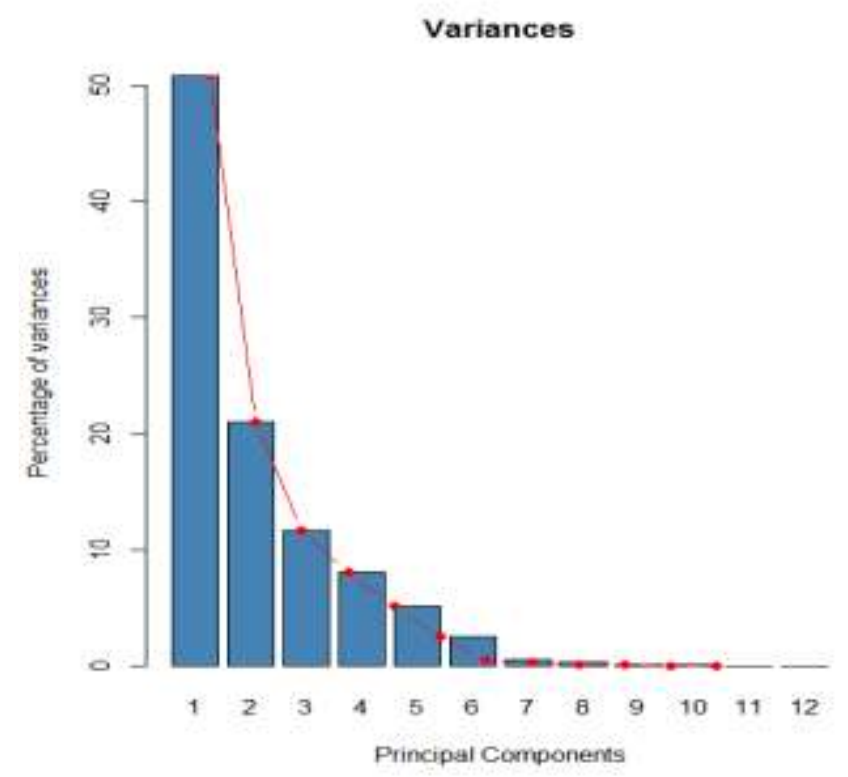

Figure 3. Variances of the Principal Components

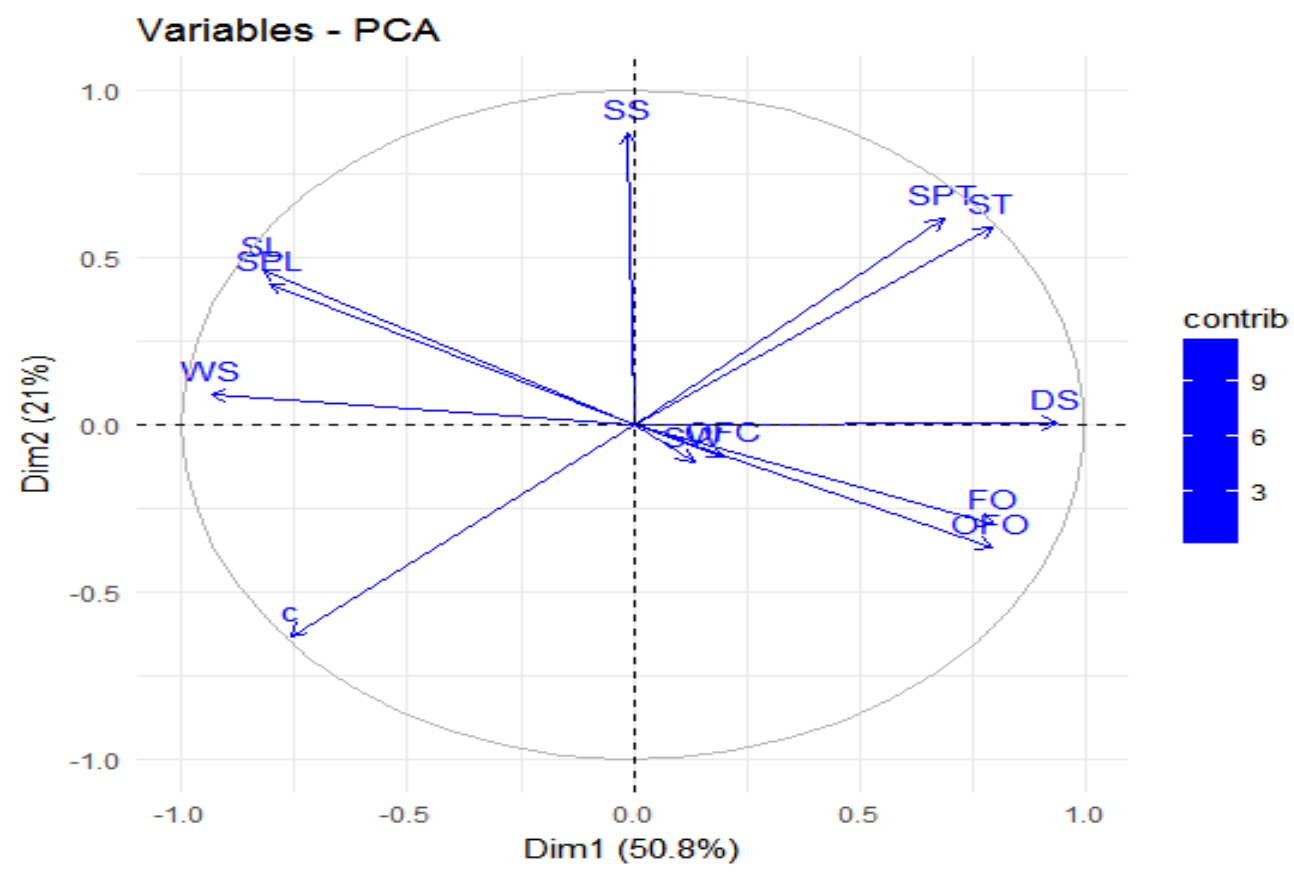

Figure 4. Relative Importance of the Features Selected by Principal Component Analysis (PCA)

After feature selection, we applied classification techniques to the selected features. We divided the datasets into two sets: one for training and one for testing. The results of the performance measures for different feature sets are shown in Figures 5-9. 


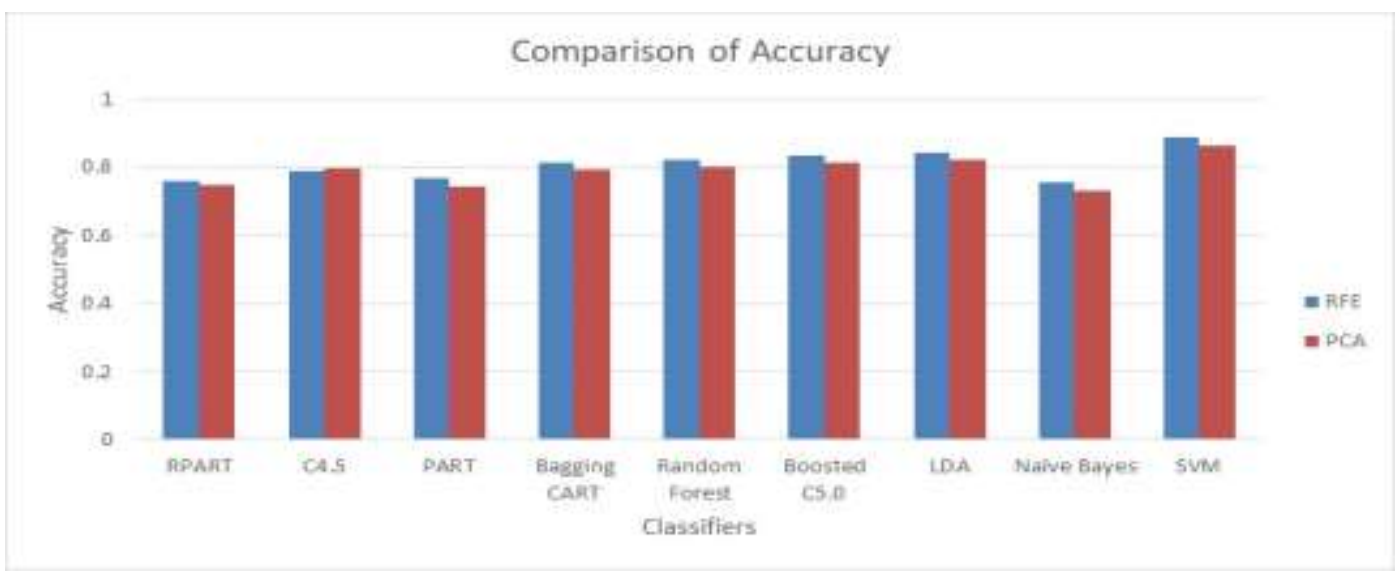

Figure 5. Accuracy of the RFE and PCA Results

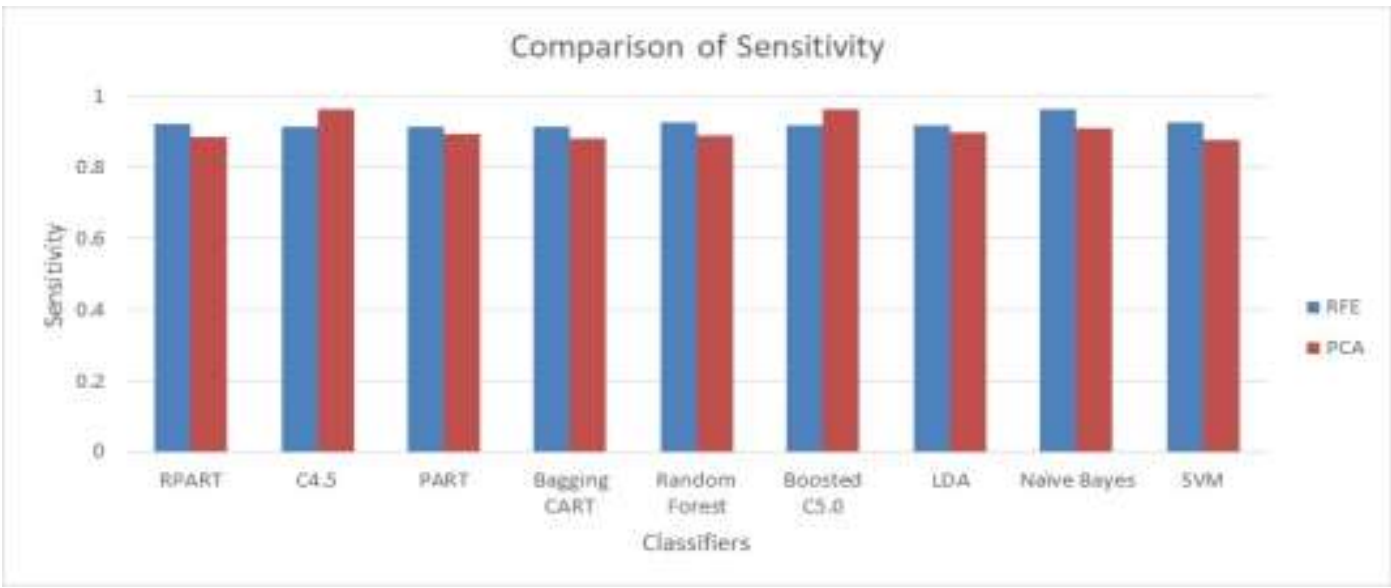

Figure 6. Comparison of the RFE and PCA Sensitivities

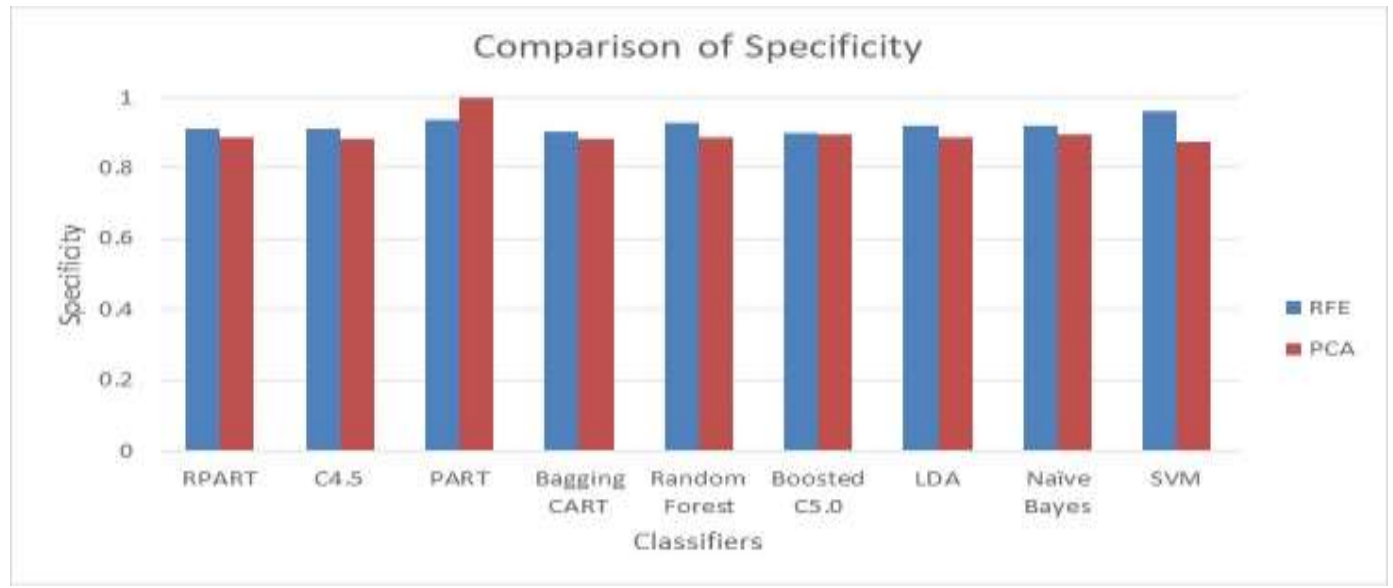

Figure 7. Comparison of the RFE and PCA Specificities

The SVM with RBF kernel function performed better with the RFE feature set than with the PCA feature set, and at a higher accuracy compared with the other classifiers (88.89\%; Figure 5). C4.5 and Boosted C5.0 produced the highest sensitivity (0.963) with PCA, whereas Naiive Bayes showed the highest sensitivity with RFE (0.963; Figure 6). PART showed the highest specificity (1) with PCA, whereas the SVM provided the highest specificity (0.963) with RFE (Figure 7). The highest PPVs were seen for PART with PCA (1) and for the SVM with RFE (0.9565; Figure 8) and the highest NPVs for 
C4.5 and Boosted C5.0 with PCA (0.9) and for Naïve Bayes with RFE (0.9333; Figure 9).

These results demonstrate that the SVM with RFE feature sets produced more accurate results than did the other classifiers with the same RFE feature sets, as well as among the same group of classifiers with PCA feature sets. We also determined that the other performance measures, including sensitivity, specificity, and NPV, performed better with PCA-based feature sets than with RFE-based feature sets using classifiers other than the SVM. Overall, most of the classifiers performed well on both feature sets.

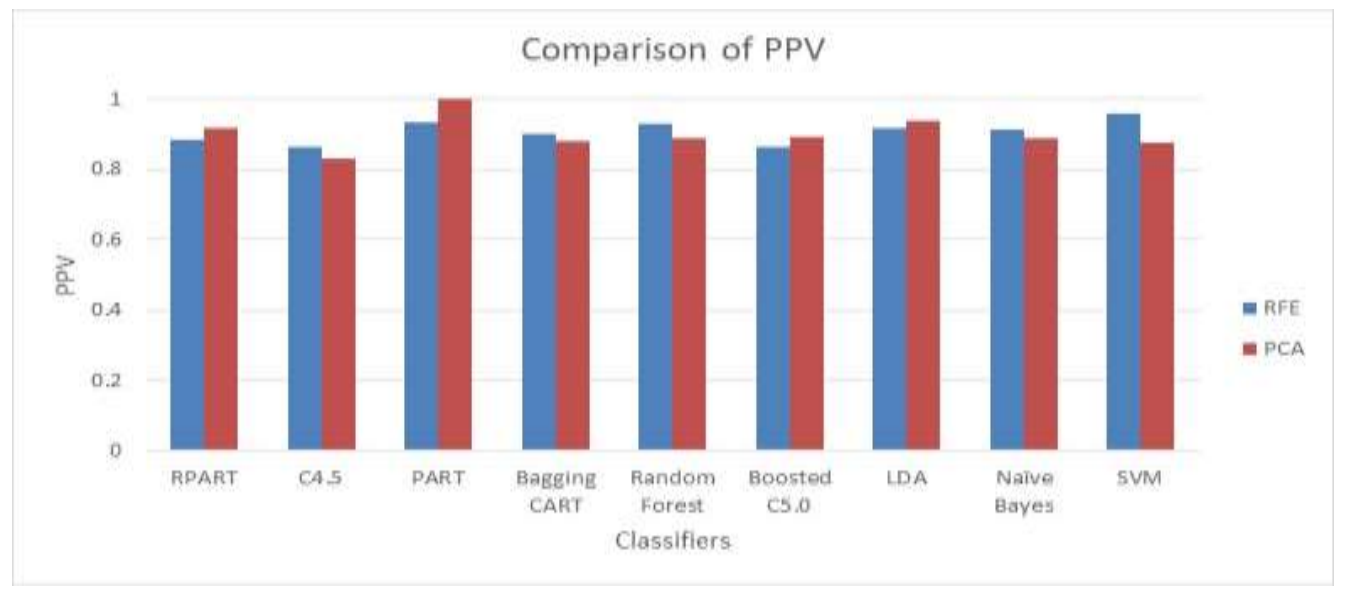

Figure 8. Positive Predictive Value (PPV) Comparison

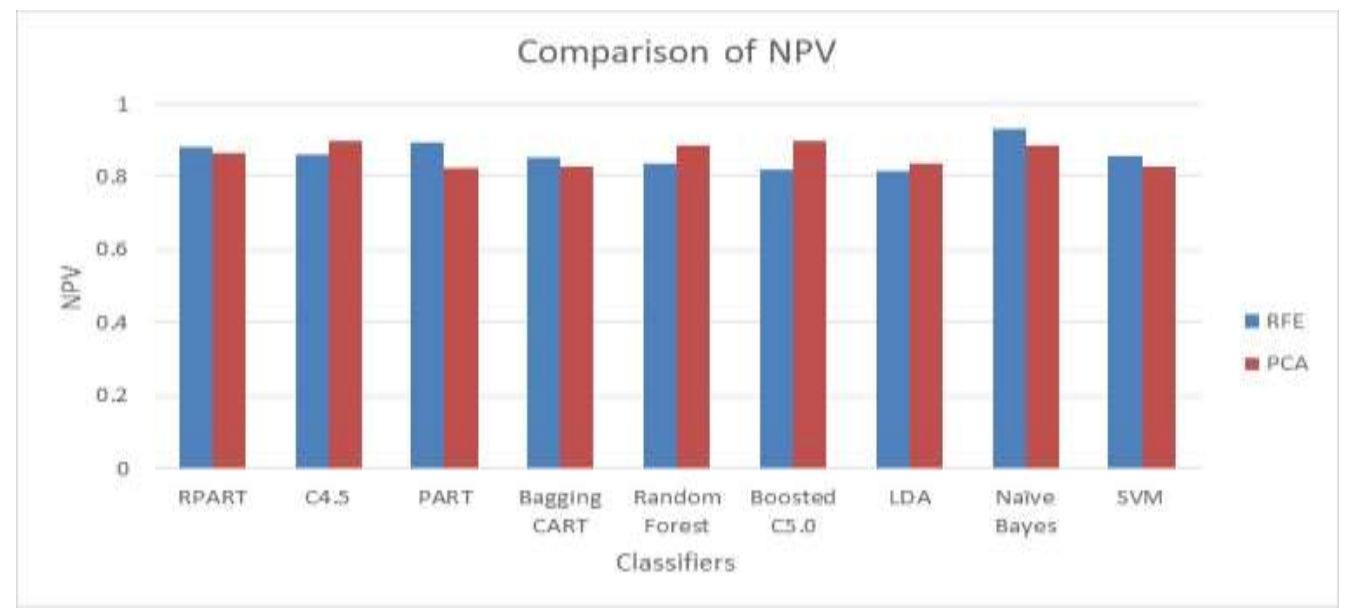

Figure 9. Negative Predictive Value (NPV) Comparison

In general, theoretical recommendations of classifiers and feature sets depend on the needs of the user. In this study, we prioritized accuracy over all other performance measures; therefore, we examined the SVM with RFE feature sets more closely than the other combinations.

\section{Conclusion}

In this study, we used linear, nonlinear, and probabilistic classifiers to distinguish PD patients from the control group using two different feature selection methods with spatiotemporal gait parameters. We obtained a maximum accuracy of $88.89 \%$ using a combination of the SVM with RBF, combined with the RFE feature sets. This analysis allowed us to explore different feature selection methods and the importance of features for improving classification performance. These results will help clinicians and doctors to automate the diagnostic and monitoring processes, such that less time and cost will be 
required for early-stage PD detection and to assess the patient's condition at different stages.

\section{Acknowledgments}

This research was supported by Basic Science Research Program through the National Research Foundation of Korea(NRF) funded by the Ministry of Science, ICT \& Future Planning (NRF-2017R1D1A3B04032905), and by the Ministry of Trade, Industry and Energy (MOTIE), KOREA, through the Education program for Creative and Industrial Convergence (Grant number N0000717). This paper is a revised and expanded version of a paper entitled "A Detailed Approach for the Development of Machine Learning based Tool for Detection and Monitoring of Parkinson's Disease from Gait Data" presented at "The Fourth Asia Workshop on IT Convergence" (AWITC), Busan, 9th February 2018.

\section{References}

[1] J. M. Beitz, "Parkinson disease: a review" Frontiers in Bioscience, S6, (2014), pp. 65-74.

[2] Y. Winter, S. Von Campenhausen, J. P. Reese, M. Balzer-Geldsetzer, K. Longo, G. Spiga, K. Boetzel, K. Eggert, W. H. Oertel, R. Dodel and P. Barone, "Costs of Parkinson's disease and antiparkinsonian pharmacotherapy: an Italian cohort study", Neurodegenerative Diseases, vol. 7, no. 6, (2010), pp. 365372.

[3] Z. X. Zhang and G. C. Román, "Worldwide occurrence of Parkinson's disease: an updated review", Neuroepidemiology, vol. 12, no. 4, (1993), pp. 195-208.

[4] D. Twelves, K. S. Perkins and C. Counsell, "Systematic review of incidence studies of Parkinson's disease", Movement disorders, vol. 18, no. 1, (2003), pp. 19-31.

[5] O. Beauchet, C. P. Launay, B. Fantino, C. Annweiler and G. Allali, "Episodic memory and executive function impairments in non-demented older adults: which are the respective and combined effects on gait performances?", Age, vol. 37, no. 4, (2015), 70.

[6] D. T. Lai, R. K. Begg and M. Palaniswami, "Computational intelligence in gait research: a perspective on current applications and future challenges", IEEE Transactions on Information Technology in Biomedicine, vol. 13, no. 5, (2009), pp. 687-702.

[7] J. C. Schlachetzki, J. Barth, F. Marxreiter, J. Gossler, Z. Kohl, S. Reinfelder, H. Gassner, K. Aminian, B. M. Eskofier, J. Winkler and J. Klucken, "Wearable sensors objectively measure gait parameters in Parkinson's disease", PloS one, vol. 12, no. 10, (2017), p.e0183989.

[8] B. Sijobert, M. Benoussaad, J. Denys, R. Pissard-Gibollet, C. Geny and C. A. Coste, "Implementation and Validation of a Stride Length Estimation Algorithm, Using a Single Basic Inertial Sensor on Healthy Subjects and Patients Suffering from Parkinson's Disease", Health, vol. 7, (2015), pp. 704-714.

[9] M. Pastorino, M. T. Arredondo, J. Cancela and S. Guillen, "Wearable sensor network for health monitoring: the case of Parkinson disease", In Journal of Physics: Conference Series, vol. 450, no. 1, (2013), p. 012055.

[10] L. Carcreff, C. N. Gerber, A. Paraschiv-Ionescu, G. De Coulon, C. J. Newman, S. Armand and K. Aminian, "What is the Best Configuration of Wearable Sensors to Measure Spatiotemporal Gait Parameters in Children with Cerebral Palsy?", Sensors, vol. 18, no. 2, (2018), pp. 394.

[11] J. L. Adams, K. Dinesh, M. Xiong, C. G. Tarolli, S. Sharma, N. Sheth, A. J. Aranyosi, W. Zhu, S. Goldenthal, K. M. Biglan and E. R. Dorsey, "Multiple Wearable Sensors in Parkinson and Huntington Disease Individuals: A Pilot Study in Clinic and at Home", Digital Biomarkers, vol. 1, no. 1, (2017), pp. 52-63.

[12] A. Suppa, A. Kita, G. Leodori, A. Zampogna, E. Nicolini, P. Lorenzi, R. Rao and F. Irrera, "l-DOPA and freezing of gait in Parkinson's disease: objective assessment through a wearable wireless system", Frontiers in neurology, vol. 8, (2017), pp. 406.

[13] T. Ilias, B. Filip, C. Radu, N. Dag, S. Marina and M. Mevludin, "Using measurements from wearable sensors for automatic scoring of Parkinson's disease motor states: Results from 7 patients", In Engineering in Medicine and Biology Society (EMBC), 2017 39th Annual International Conference of the IEEE, (2017) July, pp. 131-134.

[14] P. Prasertsung and T. Horanont, "A classification of accelerometer data to differentiate pedestrian state", In Computer Science and Engineering Conference (ICSEC), International IEEE, (2016), pp. 1-5.

[15] R. Begg and J. Kamruzzaman, "A machine learning approach for automated recognition of movement patterns using basic, kinetic and kinematic gait data", Journal of biomechanics, vol. 38, no. 3, (2005), pp. 401-408.

[16] Y. L. Hsu, P. C. Chung, W. H. Wang, M. C. Pai, C. Y. Wang, C. W. Lin, H. L. Wu and J. S. Wang, "Gait and balance analysis for patients with Alzheimer's disease using an inertial-sensor-based wearable instrument", IEEE journal of biomedical and health informatics, vol. 18, no. 6, (2014), pp. 1822-1830. 
[17] N. M. Tahir and H. H. Manap, "Parkinson Disease Gait classification based on Machine Learning Approach", Journal of Applied Sciences, vol. 12, no. 2, (2012), pp. 180-185.

[18] W. Froelich, K. Wrobel and P. Porwik, "Diagnosis of Parkinson's disease using speech samples and threshold-based classification”, Journal of Medical Imaging and Health Informatics, vol. 5, no.6, (2015), pp. 1358-1363.

[19] D. C. Li, C. W. Liu and S. C. Hu, "A fuzzy-based data transformation for feature extraction to increase classification performance with small medical data sets", Artificial Intelligence in Medicine, vol. 52, no. 1, (2011), pp. 45-52.

[20] M. Shahbakhi, D. T. Far and E. Tahami, "Speech analysis for diagnosis of Parkinson's disease using genetic algorithm and support vector machine", Journal of Biomedical Science and Engineering, vol. 7, no. 4, (2014), pp. 147-156.

[21] B. Pogorelc, Z. Bosnić and M. Gams, "Automatic recognition of gait-related health problems in the elderly using machine learning", Multimedia Tools and Applications, vol. 58, no. 2, (2012), pp. 333354.

[22] M. Kim and H. Park, "Using Tractography to Distinguish SWEDD from Parkinson's Disease Patients Based on Connectivity”, Parkinson's Disease, (2016).

[23] Y. J. Yi and Y. S. Yu, "Emergency monitoring system based on a newly-developed fall detection algorithm", Journal of information and communication convergence engineering, vol. 11, no. 3, (2013), pp. 199-206.

[24] I. H. Youn, K. Won, J. H. Youn and Scheffler, "Wearable Sensor-Based Biometric Gait Classification Algorithm Using WEKA", Journal of information and communication convergence engineering, vol. 14, no. 1, (2016), pp. 45-50.

[25] R. Prashanth and S. D. Roy, "Novel and Improved Stage Estimation in Parkinson's Disease using Clinical Scales and Machine Learning", Neurocomputing, vol. 305, (2018), pp. 78-103.

[26] E. Abdulhay, N. Arunkumar, K. Narasimhan, E. Vellaiappan and V. Venkatraman, "Gait and tremor investigation using machine learning techniques for the diagnosis of Parkinson disease", Future Generation Computer Systems, vol. 83, (2018), pp. 366-373.

[27] P. M. G. Granitto, C. Furlanello, F. Biasioli and F. Gasperi, "Recursive feature elimination with random forest for PTR-MS analysis of agroindustrial products", Chemometrics and Intelligent Laboratory Systems, vol. 83, no. 2, (2006), pp. 83-90.

[28] E. R. Hruschka and N. F. Ebecken, "Extracting rules from multilayer perceptrons in classification problems: A clustering-based approach", Neurocomputing, vol. 70, (2006), pp. 384-397.

[29] H. B. Wong and G. H. Lim, "Measures of diagnostic accuracy: sensitivity, specificity", PPV and NPV, Proceedings of Singapore healthcare, vol. 20, no. 4, (2011), pp. 316-318.

\section{Authors}

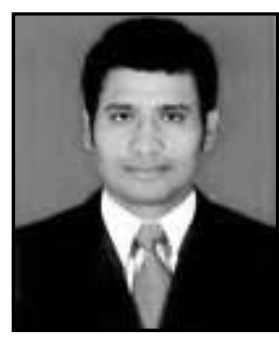

Satyabrata Aich, is working as a researcher in the field of computer engineering. He has published many research papers in journals and conferences in the realms of Machine learning, Text mining, and Supply Chain Management. His research interests are natural language processing, Machine learning, supply chain management, Text mining, Bio informatics.

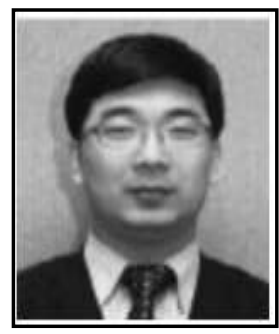

Kim Hee-Cheol, received his BSc at Department of Mathematics, MSc at Department of Computer Science in SoGang University in Korea, and $\mathrm{PhD}$ at Numerical Analysis and Computing Science, Stockholm University in Sweden in 2001. $\mathrm{He}$ is Professor at Department of Computer Engineering and Head of the Institute of. Digital Anti-aging Healthcare, Inje University in Korea. His research interests include Machine learning, Text mining, Bio Informatics. 